\title{
Fuzzy based Efficient Service Broker Policy for Cloud
}

\author{
Nazmul Islam \\ Department of Information and Communication \\ Technology \\ Mawlana Bhashani Science and Technology \\ University, Santosh, Tangail, Bangladesh
}

\author{
Sajjad Waheed \\ Department of Information and Communication \\ Technology \\ Mawlana Bhashani Science and Technology \\ University, Santosh, Tangail, Bangladesh
}

\begin{abstract}
Cloud computing is a type of internet based computing that provides shared computer processing resources, storage and data to computers on user demand. Today's time, it becomes very popular due to new facilities and technologies. It deals with large amount of data so that it is necessary to simulate the behavior of cloud in real field. So that the simulation tools as like cloud-analyst, cloudsim are commonly used. These simulators are using different load balancing policy and service brokerage strategy. My proposed service brokerage strategy increases the efficiency and minimizes the cost.
\end{abstract}

\section{Keywords}

Simulation, fuzzy, Service broker policy, cloud computing, cloud-analyst.

\section{INTRODUCTION}

Cloud computing is a large-scale spreader computing paradigm that is driven by economies of large scale, in which a pool of abstracted, virtualized, and dynamically scalable managed computing power, platforms, storage and services are provided on demand to external customers over the Internet [1], [2]. Progress is being made in cloud computing every day.

Cloud computing is designed to provide service rather than a product. Transparency is one of the main design issues of cloud computing, in which services such as computation, software, data access, and storage are provided to users without the knowledge of the physical location and configuration of the server that provides such services [3].

Given the progress of cloud services, large-scale software systems, such as social networking sites and e-commerce applications, are gaining popularity. These software systems greatly utilize cloud services to minimize costs and improve service quality to end users. Several factors affect the cost and quality of the cloud. Some of these factors are the distribution (geographic) of the user bases, the availability of the Internet infrastructure within those geographic areas, the dynamic nature of the usage patterns of the user base, and how well cloud services can be adapted or dynamically reconfigured.

Some studies have used simulation techniques to investigate the behavior of large-scale distributed systems [4], [5]. These studies have shown that cloud analysis [6] can be used for simulating this type of large-scale applications.

Cloud computing has different metrics, including fault tolerance, availability, scalability, flexibility, reduced overhead for users, performance, and on-demand services. Algorithms, policies, and methodologies are necessary to achieve high user satisfaction and resource utilization in cloud computing by ensuring an efficient and fair allocation of every computing resource. Selecting proper service broker policies and scheduling algorithms in the cloud environments of large-scale applications serves an essential function in the system performance [4], [5]. Thus, a technique must be designed to minimize the response time, cost, resource utilization, and overhead by distributing the user application workload among various data centers [7].

Driven by the preceding considerations, this study proposes and implements a new service broker policy (data center selection) based on the datacenter distance and service response time. The target of this paper is to introduce a new service brokerage strategy which is more efficient both on cost and utilization of data center.

\section{RELATED WORKS}

In cloud computing, the geographical location of the target data center has a significant influence on overall performance of cloud system because of different network issues and the location of user groups [8]. The service broker policy is responsible for routing the requests of users originating from different user groups, which are located in different geographical regions in the globe, to the data centers in cloud. The data centers are also distributed in different geographical regions.

The standard service broker policy provided by Cloud Analyst can be any of the following types:

- Service Proximity-Based Broker Policy

This policy is considered the shortest path data center. The service broker sends the request to the closest data center in consideration of network latency.

- Performance Optimization Policy

In this policy, the service broker actively monitors all data centers and sends the request to the data center that provides the best response time to the end user at the time of query.

- Dynamic Configuration Policy

In this policy, the service broker is assigned the additional job of scaling the application deployment depending on the load it is currently facing. The service broker dynamically increases or decreases VMs in the data centers according to the current processing times relative to the best processing time that has ever been achieved.

A number of studies have been conducted on the effect of service broker policy on the performance of cloud environments [4], [5], [8]. [4], [5] used CloudAnalyst to represent the performance analysis of three previous broker policies in combination with different load balancing policies for large-scale applications using different infrastructural environments. They concluded that the service broker policy affects the overall response time of the system. Although

P. M. Rekha et. at. [8] analyzed various service broker policies for selecting data centers and compared their cost, the idea of service brokering in a cloud continues to be the subject of many research works. 


\section{INTRODUCTION TO CLOUDANALYST \& FUZZY LOGIC}

\subsection{CloudAnalyst}

CloudSim enables smooth modeling, simulation, and experimenting on cloud computing infrastructure [9]. It is a platform that can be used to model data centers, service brokers, and scheduling and allocation policies of large- scale cloud platforms. CloudAnalyst [6] is built directly on top of the CloudSim toolkit.

The following are the main features of CloudAnalyst:

- Easy-to-use graphical user interface (GUI)

- Ability to define a simulation with a high degree of configurability and flexibility

- Repeatability of experiments

- Graphical output

- Use of consolidated technology and ease of extension

The following are the main components of CloudAnalyst and the function of each component:

- GUI Package. It is responsible for the GUI, for serving as the front end controller for the application, and for managing screen transitions and other user interface activities.

- Region. Six regions correspond to six continents in the world.

- User Base. This component models a group of users and generates traffic that represents the users.

- Data Center, It encapsulates a set of computing hosts or servers that are either heterogeneous or homogeneous in nature depending on their hardware configurations.

- Data Center Controller, This component controls data center activities.

- Cloudlet. It specifies a set of user requests. It contains the application ID, name of the user base as originator for routing back the responses, size of request execution commands, and input and output files.

- Internet. This component models the Internet and implements the traffic routing behavior.

- Internet Characteristics. This component is used to define the characteristics of the Internet applied during simulation.

- VM Load Balancer, This component models the load balance policy used by data centers when the serving allocation is requested.

\subsection{Fuzzy logic}

Fuzzy logic idea is similar to the human being's feeling and inference process. Unlike classical control strategy, which is a point-to-point control, fuzzy logic control is a range-to-point or range-to-range control. The output of a fuzzy controller is derived from fuzzifications of both inputs and outputs using the associated membership functions. A crisp input will be converted to the different members of the associated membership functions based on its value. From this point of view, the output of a fuzzy logic controller is based on its memberships of the different membership functions, which can be considered as a range of inputs [10].

To implement fuzzy logic technique to a real application requires the following three steps:

1. Fuzzification - convert classical data or crisp data into fuzzy data or Membership Functions (MFs)

2. Fuzzy Inference Process - combine membership functions with the control rules to derive the fuzzy output

3. Defuzzification - use different methods to calculate each associated output and put them into a table: the lookup table.
Pick up the output from the lookup table based on the current input during an application

As mentioned before, all machines can process crisp or classical data such as either ' 0 ' or ' 1 '. In order to enable machines to handle vague language input such as 'Somehow Satisfied', the crisp input and output must be converted to linguistic variables with fuzzy components.

\section{PROPOSED FUZZY BASED SERVICE BROKER POLICY}

CloudAnalyst environment has three entities: Cloud information service, service broker and data center. Cloud information service is a kind of registry that holds the information about data centers. There can be any number of data centers in the cloud environment and each data center need to be registered with a cloud information service.

As depicted in Figure 1, Service broker communicates with cloud information service and retrieves information about data centers. The Fuzzy based service broker policy routes the user base's request to the suitable data center. Data center controller and VM load balancer, involves when the user request reached a particular data center for processing. VM load balancer helps to distribute the workload among the available VMs.

The proposed fuzzy based service brokerage policy works with two main parameters datacenter distance from user base and their response efficiency. Depending on this two parameter the fuzzy method find out the nearest and most efficient Datacenter.

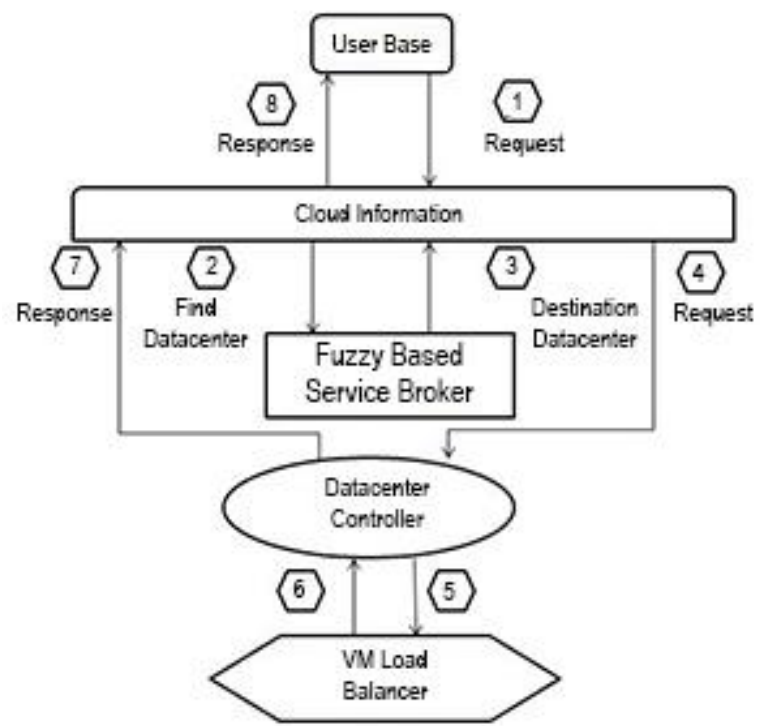

Fig 1: Request routing through fuzzy based service broker policy

During find out the targeted datacenter it is necessary to generate membership function and fuzzy rules.

To evaluate and implement the above proposed approach of fuzzy based service broker policy implementation is performed followed by the cloud and network investigation using a simulator [11].

Environment Setup: The environmental setup is performed for the Cloud using an effective simulator cloudAnalyst for investigation specific implementation. 
Fuzzy Mechanism: In our proposed design, we have emphasized on the improvement of the fuzzy based to other service brokerage policy following to the rules generation. The fuzzified service brokerage policy will be better compared to other service brokerage policy

Rule Generation: The Master Fuzzy Context (MFC) will conduct as the member function for provisional fuzzy logic to be implemented using MATLAB in this paper. The rules to be set up in the input methods are formed with distance and response time data parameters and the output methods is the nearest and efficient data center. Hence, Table 1 holds the rules for the proposed cloud to be designed.

Table 1. Set of fuzzy rules to identify efficient data center

\begin{tabular}{|l|l|l|}
\hline Distance & Response & $\begin{array}{l}\text { DC Priority } \\
\text { Value }\end{array}$ \\
\hline Very long & Too Delay & Very low \\
\hline Very long & Delay & Low \\
\hline Very long & Quick & Low \\
\hline Very long & Very Quick & Medium \\
\hline long & Too Delay & low \\
\hline long & Delay & Medium \\
\hline long & Quick & High \\
\hline long & Very Quick & High \\
\hline Sort & Too Delay & Medium \\
\hline Sort & Delay & High \\
\hline Sort & Quick & High \\
\hline Sort & Very Quick & Very High \\
\hline Very sort & Too Delay & Medium \\
\hline Very sort & Delay & High \\
\hline Very sort & Quick & Very High \\
\hline Very sort & Very Quick & Very high \\
\hline
\end{tabular}

Using membership function Distance and Response with DC Priority rules are mentioned on above table (Table 1). The approximate priority value is generated like below on simulation results. The simulation output is effective for each datacenter for each user base interaction. If there is multiple Datacenter on multiple regions it is needed to run the simulation for each datacenter.

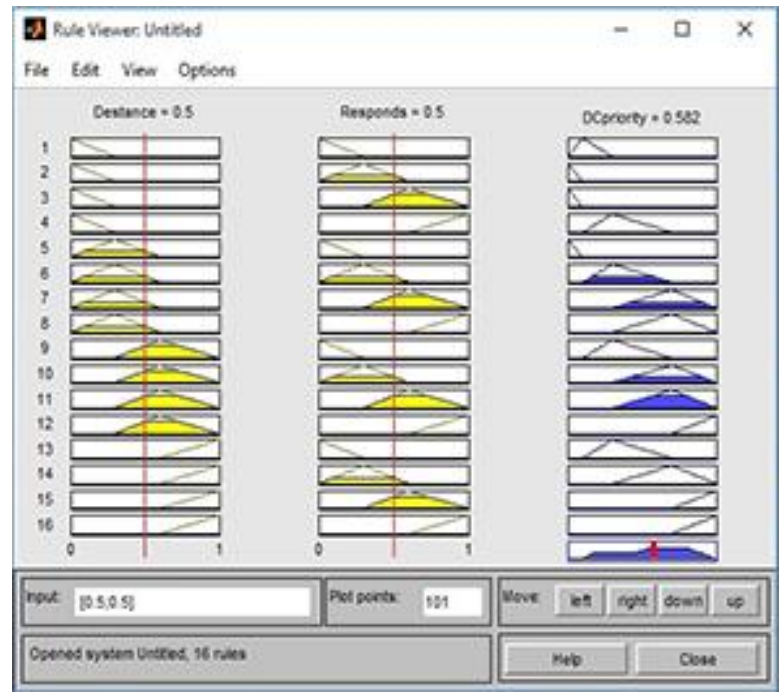

Fig 2: Distance with response time and destination observation.
From the Figure 2 it is clear to find the most closest and very quick response Datacente. The data priority value identify the datacenter priority. Using those priority result and cloudAnalyst simulator we are able to find the lest cost and efficent Datacenter to the user of the user base .

\section{SIMULATION AND RESULT ANALYSIS}

The Simulation has been done by using cloudAnalyst tool. In order to analyze different service broker policies the cloud analyst tool need to be set. One user base UB0 is used with four different datacenter DC1, DC2, DC3, DC4 and the simulation result are followed with Figure 3 and Figure 4.

To run the simulation on CloudAnalyst it is needed to get the DC priority list from fuzzy results for each datacenter along with specific user.

The Figure 3 analyzes the response of datacenter using different service brokerage policy from the CloudAnalyst simulation results. Here it is visible that on the response time of Datacenter is very similar with both closest datacenter and Fuzzy based service broker policy.

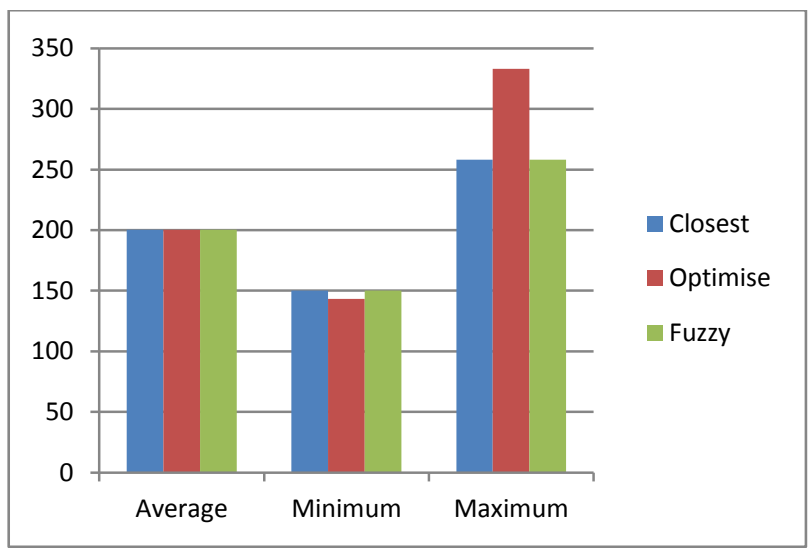

Fig 3: DC Response time on different service broker policy.

Although the fuzzy based service broker policy response time is similar to closest datacenter service broker policy but it reflects on operating cost. The Figure 4 define the cost diversity among other service broker policy.

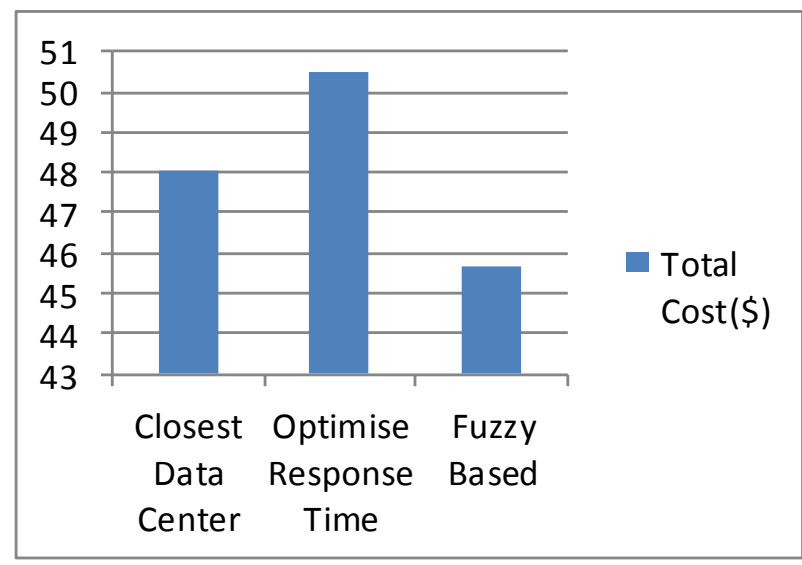

Fig 4: Cost diversity among with other service broker policy . 
From above figure it is clearly visible of costing of all the existing service brokarage policies. Closest data center service brokarage shows less cost then the optimise response time service brokarage policies and my proposed service brokarage policies shows the minimum cost from all other service brokarage plicies .So it is clear with the figure that my proposed service brokarage policy is most cost effictive service brokarage policy.

\section{CONCOLUTION}

In this paper, a newly advanced and efficient service broker policy is proposed and then implemented in cloud computing environment using CloudAnalyst simulator. The proposed policy accommodates the features of closest path and optimal response time policy. By visualizing the graphs and tables it can easily identify that the total cost efficiency is improved in comparison to the existing policies. The proposed algorithm does not include the changes occur in cloud. In future this work can be implemented in dynamic environment with more parameters. The fuzzy based load balancing policy with fuzzy based service broker policy will focus on my future work and it will be more efficient and effective.

\section{REFERENCES}

[1] Foster, I., et al. Cloud computing and grid computing 360-degree compared. In Grid Computing Environments Workshop, 2008. GCE'08. 2008. IEEE.

[2] Ahmed, M., et al., An advanced survey on cloud computing and state-of-the-art research issues. Int $\mathrm{J}$ Comput Sci Issues (IJCSI), 2012. 9.

[3] Dash, M., A. Mahapatra, and N.R. Chakraborty, Cost Effective Selection of Data Center in Cloud Environment. International Journal on Advanced Computer Theory and Engineering (IJACTE), 2013. Volume-2(Issue-1): p. pp 2319 - 2526.

[4] Wickremasinghe, B. and R. Buyya, CloudAnalyst: A CloudSim-based Tool for Modelling and Analysis of
Large Scale Cloud Computing Environments. MEDC Project Report, 2009. 22(6).

[5] Bala, M., Performance Evaluation of Large Scaled Applications using Different Load Balancing Tactics in Cloud Computing. International Journal of Computer Applications, 2013. 76(No.14): p. pg 17-22.

[6] Wickremasinghe, B., R.N. Calheiros, and R. Buyya. Cloudanalyst: A cloudsim-based visual modeller for analysing cloud computing environments and applications. in Advanced Information Networking and Applications (AINA), 2010 24th IEEE International Conference on. 2010. IEEE

[7] Zia, A. and M. Khan, A Scheme to Reduce Response Time in Cloud Computing Environment. International Journal of Modern Education \& Computer Science, 2013. 5(6).

[8] Dakshayini, R.P.M.a.M., Service Broker Routing Polices in Cloud Environment: A SurveyInternational Journal of Advances in Engineering \& Technology (IJAET) 2014. Volume 6(Issue 6): p. pp. 2717-2723.

[9] Calheiros, R.N., et al., CloudSim: a toolkit for modeling and simulation of cloud computing environments and evaluation of resource provisioning algorithms. Software: Practice and Experience, 2011. 41(1): p. 2350 .

[10] Y. Bai,D. Wang, " Fundamentals of Fuzzy Logic Control - Fuzzy Sets, Fuzzy Rules and Defuzzifications", Journal of advance Fuzzy Logic Technologiesin Industrial Application, 2006, xxv,334p.

[11] A.I Swapna, Md. H Rahman, Md. Akramuzzaman, "Performance Evaluation of Fuzzy Integrated Firewall Model for Hybrid Cloud Based on Packet Utilization", Journal on cloud computing, 2016 IEEE 978-1-46738515-2. 